\section{ONOMÁVAEIN}

Revista semestral de lingüística, filología y traducción

\title{
Dificultades metodológicas de los estudiantes sinohablantes de español como lengua extranjera
}

Methodological Difficulties of the Chinese Speaking Students of Spanish as a Foreign Language

\section{Michel René Bega González}

Universidad de Las Palmas de Gran Canaria

España

\section{(C) $($ (i) $\ominus$}

Michel Bega: Universidad de Las Palmas de Gran Canaria, España.

| Correo electrónico: omashte_@hotmail.com 


\section{Resumen}

El análisis de los errores y las dificultades que sufre el colectivo sinohablante de ELE no solo comprende la clasificación de errores de índole fonética, fonológica o gramatical. Existe otro espacio relevante de investigación que se relaciona directamente con los parámetros lingüísticos de análisis de errores y con su emisión. Hablamos aquí de las dificultades que aparecen a raíz del empleo de una metodología concreta. El presente artículo es fruto del análisis de estas dificultades llevado a cabo en los individuos pertenecientes a la Universidad Normal de Changchun, en la República Popular China. Sus resultados reflejan que la metodología de enseñanza aplicada en este particular suscita que los aprendices cometan determinados errores y tengan dificultades comunicativas.

Palabras clave: ELE; China; sinohablantes; problemas; metodología.

\section{Abstract}

The analysis of the errors and difficulties suffered by the Chinese speakers who learn Spanish as a Foreign Language is not only based on the classification of phonetic, phonologic or grammar mistakes. There is another relevant research path which is directly related to the linguistic parameters of error analysis and the origin of these errors. We hereby talk about the difficulties that appear because of using a concrete teaching method. The present article is the result of the analysis of these difficulties on the students who belong to the Changchun Normal University, in Peoples' Republic of China. Its results reflect that the teaching methods applied in this context cause errors and communicative problems in the apprentices.

Keywords: Chinese speakers; Spanish; problems; methods. 


\section{Introducción}

Podemos afirmar que, desde hace relativamente poco tiempo, hemos estado siendo testigos de un fenómeno en el que cada vez más estudiantes pertenecientes a la República Popular China quieren, por diversos motivos o circunstancias particulares, aprender nuestra lengua. Como resultado de este creciente número de sinohablantes y de la consecuente demanda que el colectivo docente de ELE y EL2 está experimentando, los investigadores expertos en la materia han realizado estudios que nos hacen comprender cuál es la realidad que caracteriza a este numeroso colectivo y le diferencia del resto de, por ejemplo, aprendices asiáticos. Podemos citar, como muestras relevantes, las aportaciones de Sánchez Griñán (2009) en su estudio de la cultura china de aprendizaje, o de Yu (2001) en lo referente a la implantación de un sistema comunicativo exitoso en este entorno, fuertemente caracterizado por la presencia incuestionable del sistema didáctico estructuralista.

Hasta la fecha, podemos percatarnos de que, entre las áreas de investigación más estudiadas, se encuentran el entorno metodológico de enseñanza en el individuo chino y el error del aprendiz sinohablante durante el aprendizaje de español como lengua extranjera o como segunda lengua.

\section{El enfoque empleado en la enseñanza de ELE en China y la metodología comu- nicativa}

La enseñanza en la República Popular China se puede sintetizar, hasta ahora, en una palabra: estructuralismo - enseñanzas centradas en la adquisición de la lengua a través del estudio de las estructuras léxicas y gramaticales, haciendo uso de la memoria y carente de contextos verosímiles. Ya sea el aprendizaje de su propia lengua como la metodología que se emplea para cualquier otro tipo de materias, todo confluye en un solo proceso de sistematización y memorización. Esta costumbre supone la principal influencia a la hora de enseñar español como lengua extranjera en este lugar, ya que, al sustentarnos en un enfoque eminentemente comunicativo ${ }^{1}$, no solo romperemos los esquemas de los aprendientes, sino que, además, nosotros mismos nos veremos obligados a sufrir muchos procesos de adaptación. Si bien, entre los propósitos que se buscan con el empleo del enfoque comunicativo en la enseñanza, se encuentran el establecimiento de un vínculo entre las actividades del alumno y el mundo real (Hu, 2002) en lugar de ejercitar la lengua en una situación carente de contexto, y el de conferir mayor responsabilidad a los alumnos en su propio aprendizaje (García Santana-Cecilia).

Sánchez Griñán (2006) nos explica que el factor principal por el cual el enfoque comunicativo no funciona en las aulas chinas de ELE es la "cuestión burocrática" de que existiera una asignatura Ilamada "español normal" (dedicada exclusivamente a la adquisición de las competencias léxica y gramatical), impartida por profesorado exclusivamente chino, cuya importancia fuera mayor que la de otras materias. De este hecho se deriva la realidad de que el alumnado chino no acepta otro enfoque de enseñanza y no consigue comprender qué objetivos persigue el profesor con este enfoque. Para el aprendiente, el enfoque comunicativo no supone más que una "decoración" del estudio memorístico tradicional, en lugar de lo que realmente pretende. Nuestro enfoque pedagógico queda, en esta fase, desechado por el estudiante y, en otras palabras, por el propio sistema en la enseñanza de ELE en este territorio.

Janice Penner (1995: 2) también nos muestra el rechazo total de los sinohablantes en lo que respecta a la metodología comunicativa. Nos

1 Cuando decimos "eminentemente comunicativo", nos referimos a que el enfoque metodológico aplicado es, en mayor medida, comunicativo, pero nunca deja de ser ecléctico, pues el eclecticismo rompe las limitaciones de cada enfoque (Kumaravadivelu, 1994). 
introduce también cuestiones como la preparación del profesorado en China y los diferentes focos de relevancia - por ejemplo, no se busca una metodología innovadora, sino que el docente domine un amplio espectro de contenidos (por tanto no nos centramos aquí en la difusión de esos contenidos, sino en su mera posesión).

Yang Xu (2010: 161) muestra, desde el punto de vista de la enseñanza del inglés como lengua extranjera, su preocupación por el rechazo de este enfoque en la República Popular China, y concluye con que el sistema educativo chino, el tipo de exámenes a los que se enfrentan los aprendientes, los hábitos de enseñanza y aprendizaje, y otros factores derivados de estas realidades, concatenan este rechazo. Explica también que el profesorado debe hacer todo lo posible por combinar ambos enfoques y crear así un marco de enseñanza ecléctico.

Por su parte, Marianne Celce-Murcia y Elite Olshtain (2000) nos advierten de que, para lograr una buena recepción del enfoque comunicativo, el profesor extranjero debe percatarse de incorporar contenidos a su enseñanza que se relacionen con la pragmática textual, con el propósito de que los alumnos logren aproximarse a un modelo comunicativo en el aula y logren entenderlo y hacerlo suyo. El docente extranjero también debe tener en cuenta, a la hora de enseñar bajo estos parámetros, instruir al aprendiente chino de tal forma que este distinga entre lo gramaticalmente correcto y lo adecuado, apropiado o aceptable dentro de los parámetros de comunicación. Todo ello forma parte de una buena competencia comunicativa (Cenoz Iragui, 2004: 10).

En cuanto a la implantación de una metodología de enseñanza-aprendizaje occidental, podemos decir que no hay un acuerdo universal. Al margen de las diferentes preferencias ante utilizar un enfoque comunicativo, ecléctico o estructuralista, parece ser que muchos autores coinciden en que, sea como fuere el enfoque más adecuado, lo que prima en su implantación es que este logre adaptarse a las necesidades del aprendiente (Aguirre Beltrán, 2004: 646); no obstante, en el ámbito sinohablante tiende a generalizarse la idea de que todos los estudiantes chinos muestran predilección por un aprendizaje pasivo y conductista (Wang y Moore, 2007), cuando la realidad difiere tanto personal como geográficamente. Además de esto, también debemos aprender de los errores cometidos en el ámbito de la enseñanza de inglés, pues este es un campo más longevo y que inició su desarrollo con anterioridad al de la enseñanza de ELE. Los diferentes enfoques metodológicos que se han ido aplicando a lo largo de la historia de la enseñanza han mostrado aspectos positivos y deficiencias en el sistema educativo de las cuales podemos aprender en la actualidad para no cometer los mismos errores (Martín Martín, 2003).

\subsection{La presencia del entorno y su in- fluencia en la metodología de enseñan- za en los sinohablantes}

Cuando hablamos de una metodología, ya sea estructuralista, comunicativa o de cualquier otra naturaleza, hablamos también de los elementos que la conforman, los cuales deben estar siempre presentes si este enfoque se quiere implantar con éxito. Atendiendo a los criterios expuestos por Ruiz Campillo (1994), Lozano López (1994) y Fruns Giménez, en un método de enseñanza se encuentran los siguientes factores: el enfoque (teorías sobre la lengua y su aprendizaje), el diseño (objetivos, actividades y materiales) y el procedimiento (prácticas y conductas de clase).

Debemos saber que cada aprendiente posee su —digámoslo de esta manera_- "propia dimensión". Con esto queremos decir que a cada sujeto le afecta su propio entorno personal, social, el espacio en el que se mueve, $y$, dentro del aprendizaje de otras lenguas, estos factores también se reflejan en el individuo, sobre todo en sus mecanismos de aprendizaje. Por ejemplo, el hecho de que un individuo sinohablante no conozca otro medio más que la memorización para aprender conceptos refleja una influencia 
que ha recibido de su entorno y del contexto social en el que se encuentra. De la misma forma sucede esto con los estudiantes que emplean otra metodología de aprendizaje diferente, pues también han sido influidos por su entorno. En otras palabras, el estudiante de una segunda lengua ve afectado su aprendizaje por factores de mecanismo interno, por los factores externos y por los conocimientos del individuo (Ellis, 1994; Alcoholado, 2011). Debemos destacar aquí que la base cultural del sujeto influye en sus destrezas de aprendizaje (Anglada, 2011).

Además de los efectos positivos que el enfoque comunicativo posee cuando logra implantarse en el alumnado, surgen también las nociones de colegialidad y de la educación en valores. La colegialidad (De Miguel Díaz, 2004: 160) se define como el concepto en el que no solamente participa en la enseñanza un docente (es individualista), sino que todos los profesores de una misma área trabajan de forma colaborativa con el propósito de crear, de manera consensuada, el programa metodológico que más apto resulte para los aprendientes. Esto es, "armar un contenido coherente y con continuidad" (Zabalza Beraza, 2007). El hecho de que todos los docentes de un mismo centro converjan enriquece sobremanera el aprendizaje del alumno, y les presenta la lengua de una mejor manera (Hawkins, 1984). En lo que respecta a la enseñanza en valores, el enfoque comunicativo puede hacer del profesor un educador en valores y pasar de lo meramente didáctico al desarrollo y crecimiento personal de los individuos.

\subsection{La adaptación del enfoque comuni- cativo en la enseñanza a los aprendices sinohablantes}

Cuando aplicamos una metodología comunicativa, debemos tener en cuenta, por una parte, que la comunicación no es el único propósito relevante para la pedagogía de las segundas lenguas -esto significa, en otras palabras, que no podemos fundamentarnos en este enfoque como si de una panacea se tratara (Swan, 1985; Canale, 2012) - y, por otra, las aportaciones de Littlewood (1981) en lo concerniente a la tipología de las actividades presentadas en el aula. Al principio, Littlewood recomienda emplear actividades controladas que presenten estructuras. Esta fase, al ser la inicial, se considera necesaria, pese a que se aleja de la metodología eminente que queremos implantar; no obstante, con posterioridad se procedería a presentar actividades que contemplaran la resolución de problemas desde una perspectiva comunicativa.

Alrededor de la dificultad que suscita la adaptación de un enfoque comunicativo en la enseñanza a sinohablantes surgen varias preguntas: ¿por qué el aprendizaje de estos alumnos es puramente memorístico?, ¿cuáles son los rasgos considerados negativos de este proceder?, ¿existen factores positivos que podamos extraer de esta metodología desde el punto de vista de la enseñanza de ELE?, ¿hay soluciones prácticas a los problemas que puedan derivarse de esta praxis?

La respuesta a la primera pregunta podemos encontrarla en la lengua china. El mandarín es un idioma muy diferente al nuestro porque está compuesto por miles de caracteres que designan realidades. Estos caracteres podemos dividirlos en dos grandes grupos: los caracteres pictográficos y los caracteres ideográficos. Los caracteres pertenecientes al primer grupo son aquellos que se originan a partir de imágenes y son los más antiguos. Un ejemplo es el carácter Iuna (月), cuyo origen se encuentra en la representación gráfica de la luna que ha ido evolucionando hasta convertirse en este carácter. En el segundo tipo se encuentran aquellos caracteres que están compuestos por dos o más caracteres pictográficos. Un ejemplo que lo ilustra es ver/ mirar (看), que se compone de mano arriba (手) y ojo (目).

Para aprender estos caracteres es necesario el desarrollo y el ejercicio continuo de la memoria (Alcoholado, 2012), ya que no existe ninguna otra 
regla para aprenderlos. El aprendiente de chino debe memorizar, en primer lugar, los caracteres esenciales y, después, todos los demás, para relacionarlos con su significado al tiempo que adquieren nuevos conceptos. Cuando los aprendientes de chino extranjeros comienzan a estudiar este idioma, no solamente deben memorizar estos aspectos gráficos, sino que, además, deben relacionarlos con las realidades a las que hacen referencia y con los cuatro tonos del mandarín (cada carácter lleva, en su pronunciación, un tono, clave para diferenciarse de otros caracteres con la misma vocalización). Además de todo esto, el primer paso es aprender el alfabeto pinyin, creado para emular los sonidos del chino.

Los propios chinos memorizan estos caracteres durante sus principales etapas académicas, por este motivo se acostumbran a ello y rechazan de forma automática otro tipo de método de aprendizaje, ya que solo han desarrollado uno -el cual, a diferencia del comunicativo, es estático, tiene un carácter absoluto e intrapersonal, no interpersonal (Cenoz Iragui, 2012). Este mismo método lo aplican a todas las demás materias, ya sean lingüísticas o de otra índole. Tratan de memorizarlo todo. En particular, el español también lo aprenden de esta manera. Los profesores locales les obligan a memorizar listas de palabras cada día o cada semana. Si esto no ocurre, los propios alumnos las elaborarán para memorizarlas con posterioridad.

Los defectos de este proceder son muy notables. En primer lugar, los alumnos no adquieren las competencias necesarias para aplicar lo que aprenden; es decir, podrán memorizar todas las conjugaciones verbales y recitar largas listas de palabras, pero no les será fácil aplicarlas al contexto. Este problema lo podemos encontrar en la mayoría de los alumnos que estudian el Grado de Español. En los exámenes, pueden rellenar con éxito los huecos en los que se les pide que introduzcan las palabras. Incluso pueden aprobar, con mayor o menor acierto, los ejercicios relacionados con la redacción y el desarrollo de un tema concreto. Ahora bien, al salir a la calle, o al hablar en clase de algo que no esté relacionado con los contenidos del manual, hay un silencio absoluto. Cuando por fin se deciden a hablar, no son capaces de relacionar los contenidos lingüísticos y gramaticales adecuadamente, por lo que, aunque no sea ininteligible el discurso, las carencias son preocupantemente evidentes.

Dadas estas circunstancias, y dado que tanto los manuales empleados como las actividades que se realizan en estas aulas son forzadas (Sans) y no contemplan relación alguna con las situaciones comunicativas verosímiles, podemos decir que la implantación de un enfoque metodológico eminentemente comunicativo ayudaría al alumno a reparar los daños mencionados y, además, propiciaría que este no solamente contribuyera a la adquisición de sus conocimientos, sino también a la creación de expectativas acerca del aprendizaje de la lengua (Breen y Candlin, 1980).

El hecho de que los alumnos no hablen libremente cuando se les pregunta responde a otro factor que también está relacionado con este tipo de metodología, pero solamente en la enseñanza, no en el aprendizaje. Cuando un profesor extranjero lanza una pregunta al aire, ningún alumno quiere responder no por miedo a errar — también perceptible-, sino por temor a intervenir en un momento inadecuado. Por esta razón, muchas veces el profesorado extranjero pregunta nombre por nombre, ya que muchas veces se han producido situaciones incómodas y hasta de tensión mutua.

\subsection{Consideraciones beneficiosas para el colectivo sinohablante derivadas del trasfondo educativo chino}

Tradicionalmente, en China, el alumno debía escuchar al docente en todo momento, y no podía alzar la voz o responder si el profesor le preguntaba directamente. Hoy, aún podemos encontrar esta realidad, agravada con una metodología en la que el alumno es un ser recep- 
tivo que no emite respuesta alguna. Lo único que se espera de ellos durante las sesiones es que asimilen todos los conceptos que se les han transmitido, y que, a su vez, no se cuestione la información de ninguna manera. El hecho de no cuestionarse nada surge de la percepción subjetiva de que "lo que el profesor o el manual dice es una verdad absoluta". Un hecho que podemos encontrar comúnmente es el de la incuestionabilidad de los manuales locales. Aunque esta realidad ya está cambiando, por lo general el alumno no solamente no cuestionará los contenidos de los anuales locales, sino que, además, sí lo hará con respecto a la información transmitida por el profesorado foráneo.

Pese a todo lo negativo que conlleva esta metodología tan particular, podemos extraer rasgos positivos de ella. Está claro que no podremos cambiar el proceder del aprendiente en un corto periodo de tiempo (ni debemos forzarle a ello). Ante todo, el alumno es libre de escoger la metodología que más le convenga para adquirir el conocimiento. Lo positivo que podemos extraer es que, gracias a la capacidad de memorización presente en este colectivo, el vocabulario y las conjugaciones verbales no serán un problema. Muchas reglas gramaticales tampoco supondrán un escollo en el aprendizaje. No son muchas las ventajas en comparación con los inconvenientes que supone esta metodología, pero, aunque sean pocas, deberemos sacar provecho de ellas y combinar estos rasgos con nuestra propia metodología.

Como soluciones prácticas, podemos mantener, en primer lugar, estas características que derivan de la memorización. Las tablas con vocabulario al final de cada unidad suponen una ventaja que no podríamos hallar en otros aprendientes. Es más, debemos recordar que lo que a nosotros nos supone una pesadilla a otras personas les supone un alivio. Debemos comprender que, para el colectivo chino, el uso de la memoria es esencial. Si entendemos esto y sacamos partido de ello, llegaremos a la conclusión de que, sea cual sea la metodología que queramos emplear, jamás deberemos prescindir del uso de la memoria. Recordemos que estos sujetos han tenido la necesidad de utilizar la memoria durante todas las etapas de su vida. El mismo aprendizaje de su lengua y de los caracteres que la componen es un ejemplo de cómo funciona la mente de estos aprendientes (Sánchez Griñán, 2008). Con respecto a la aplicación de la metodología ecléctica, los problemas derivados del enfoque comunicativo se deberán ir resolviendo poco a poco, introduciendo en todo momento actividades de expresión oral ajenas a los manuales y aplicables a una situación real y verosímil. Esta etapa es la más lenta y tortuosa, porque, en primer lugar, nuestros alumnos deberán darse cuenta de que no están en un contexto tradicional de enseñanza, sino que están en una situación relajada y de libre participación. Cuando el alumno comience a adquirir esa confianza con el profesor y poco a poco se vaya acostumbrando a esta dinámica, se apreciará mejoría. Otra solución es el juego de rol, en el que el alumno se verá forzado a interpretar una escena. Aquí nos daremos cuenta de que también será importante estimular su inteligencia emocional y hacer que el miedo a hablar en público desaparezca.

En resumen, para solucionar estas dificultades, deberemos transformar muchas exigencias en actividades con cierto componente lúdico, para que el proceso de aprendizaje sea ameno y no suponga un esfuerzo titánico para el alumno chino; asimismo, este proceso de aprendizaje (comunicativo) debe ser reflejo de un entorno natural (Sánchez, 1997). Aunque pueda darles la sensación de que no están aprendiendo, debemos hacerles ver que sus progresos son evidentes; asimismo, debemos explicarles que ambos métodos son perfectamente compatibles y necesarios en cuanto a la adquisición de la lengua extranjera.

Como conclusión a nuestro análisis acerca de la metodología de enseñanza en China y de la repercusión que tiene el enfoque comunicativo 
en los alumnos chinos, consideramos necesario destacar que, aunque en un principio sea difícil, podemos lograr que la implantación de una metodología comunicativa europea sea fructífera y ofrezca buenos resultados. Lo que debemos conseguir para ello es que el alumno comprenda la finalidad de este enfoque. Para ello, el aprendiente deberá trabajar en dos dimensiones, que son la reflexión del desarrollo temático y la resolución de problemas (Cáceres Lorenzo y Santana Alvarado, 2014). Estas dimensiones se relacionan con los contenidos conceptuales y su aplicación (saber y saber hacer) y con la autonomía del individuo y sus actitudes (saber aprender y saber estar). Si el aprendiente se implica en el proceso mismo de enseñanza-aprendizaje, se logrará un éxito de la implantación del enfoque comunicativo europeo en su adquisición de conocimientos.

\subsection{El enfoque por tareas}

Uno de los aspectos más relevantes que debemos tratar al contemplar las dificultades de aprendizaje de los sinohablantes es el del enfoque por tareas. Este método es el resultado de un programa de aprendizaje de lenguas fundamentado en actividades que, en lugar de incidir en la utilización de las estructuras sintácticas, se centra en fomentar el uso de la lengua ${ }^{2}$. El objetivo que se propone este enfoque es el de fomentar el aprendizaje de la lengua mediante su uso real en el aula, y no exclusivamente a través de la manipulación de unidades.

El origen de este enfoque se remota al mundo anglosajón, aproximadamente en el año 1990. Fue el resultado de la evolución de los enfoques comunicativos de Breen (1987), Nunan (1989) y Candlin (1990). El enfoque por tareas se fundamenta en distinguir los contenidos necesarios para que se produzca la comunicación y sus procesos. La comunicación aquí no es solamente la codificación y descodificación de mensajes mediante las estructuras lingüísticas, sino también la adecuada interpretación del sentido. En esta fase aparece el contexto como elemento primordial para este enfoque.

La teoría del enfoque por tareas se materializa en una serie de procedimientos que engloban la programación y la realización de actividades en el aula de ELE. La programación es la fase en la que el alumnado desarrolla un inventario en el que figuren las estructuras morfosintácticas, el vocabulario, etc. La realización es la unificación entre los procesos de uso de la lengua con los del aprendizaje. Se aprende la lengua extranjera mediante su uso en diferentes contextos verosímiles, que van desde las situaciones reales hasta los contextos en los que el estudiante demanda un aprendizaje necesario.

El enfoque por tareas es un concepto antecesor con respecto a lo que conocemos como enfoque comunicativo (CVC, 2015). Si atendemos al marco teórico de dicho enfoque, observamos que su aplicación metodológica consiste en la combinación del enfoque por tareas y del enfoque nocional-funcional. Cada una de estas dos entidades contempla, a su vez, algunos aspectos de relevancia. La figura 1 refleja los mencionados enfoques y sus características (Vacas Hermida, 2004).

Mediante el análisis de los manuales de español de los que disponen los aprendices sinohablantes, y también mediante la aplicación inicial del método comunicativo en la enseñanza de ELE a este sector, se puede observar que, pese al arraigado método estructural tanto en la República Popular China como en el resto del continente asiático, los alumnos han empezado a responder adecuadamente, aunque con lentitud, ante este método que, en lugar de suponer una dificultad metodológica, se debería mostrar

2 De acuerdo con el Diccionario de términos clave de ELE (2014), con el uso de la lengua se hace referencia a la práctica social cuya principal función es la comunicación. La lengua es el objeto de estudio del análisis del discurso y de la pragmática, entre otras disciplinas. 


\section{FIGURA 1}

Esquema conceptual del enfoque comunicativo en la enseñanza

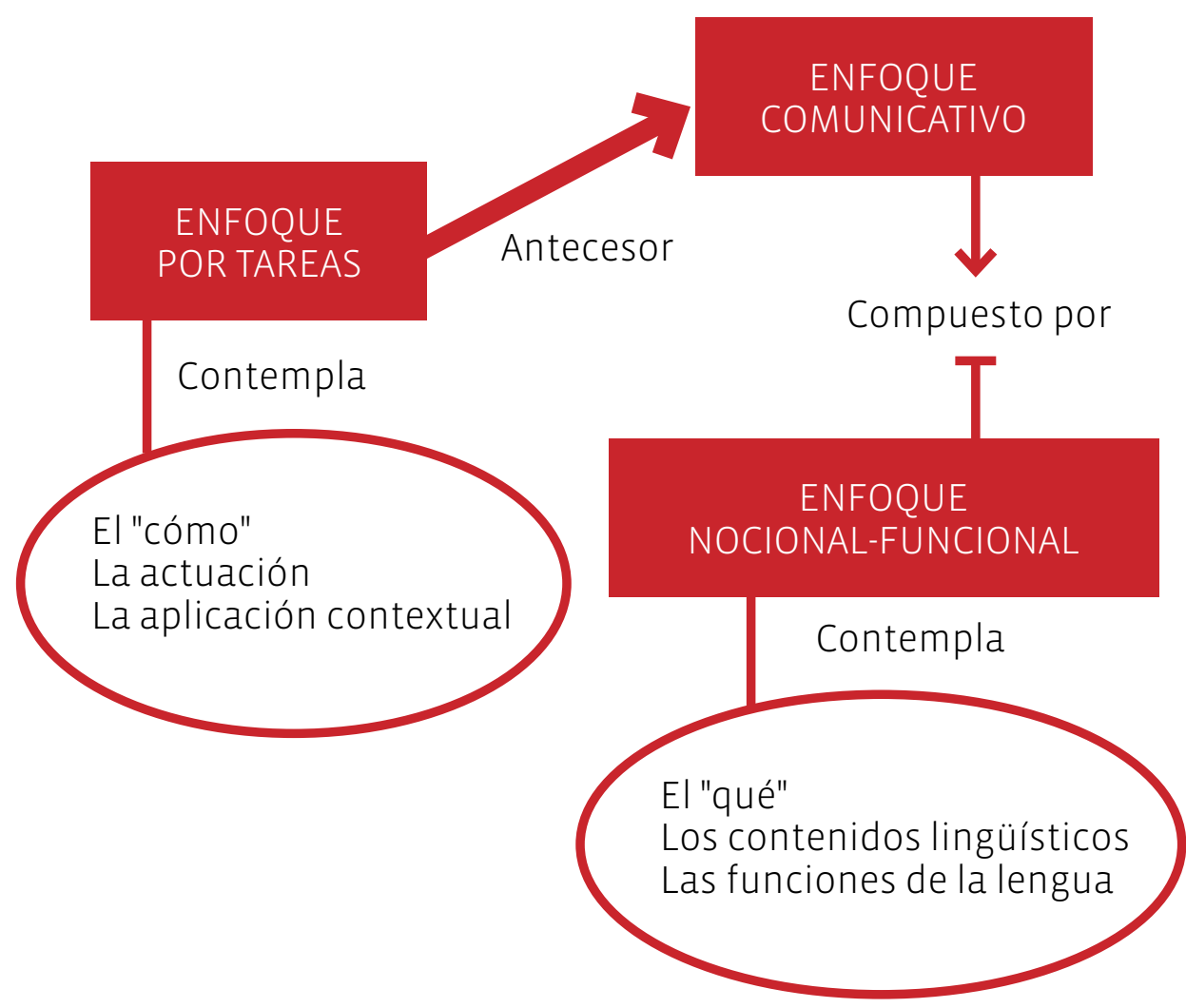

Fuente: Vacas Hermida, Asunción. “El enfoque por tareas”, Tierra de Nadie, n. ㅇ 9, 2004.

como un enfoque imprescindible e indiscutiblemente útil sobre el que sustentarse para aprender español de forma efectiva.

\section{La adaptación metodológica en los manuales ELE para sinohablantes y su repercusión en el proceso de enseñan- za/aprendizaje}

Los manuales de español como lengua extranjera destinados a los aprendientes de habla china podríamos dividirlos en dos grandes grupos. En el primero, se encuentran todos los manuales producidos en el territorio chino y, en el segundo grupo, todos aquellos producidos en España. En este particular, es necesario destacar la ausencia de Hispanoamérica debido a que la práctica totalidad de los manuales selecciona- dos por estas instituciones proceden de España.

Entre los manuales pertenecientes al primer sector, se encuentra un favorito, utilizado por todas las universidades chinas para enseñar español. Este libro, anteriormente mencionado, es Español Moderno, de la editorial Foreign Language Teaching and Research Press. Esta serie de libros se ha convertido en la referencia absoluta para profesores y alumnos. Dada la reconocida fama del autor y la consecuente fiabilidad que en un principio ofrecen los contenidos originales y las actividades relacionadas con la gramática, estas "biblias" se han convertido en una herramienta de información incuestionable.

Durante la época en la que estos manuales fueron elaborados, sus contenidos resultaron de gran ayuda y empezaron a iluminar un futu- 
ro esperanzador en la enseñanza del español en la República Popular China. Por supuesto, no se discute en absoluto la relevancia y la utilidad de estos ejemplares; sin embargo, el tiempo y la consiguiente evolución lingüística no han pasado en balde para esta vieja gloria didáctica. En otras palabras, estos manuales han quedado, hoy, obsoletos.

Además de los errores que poseen estos libros (como cualquier otro manual de español, pues recordemos que es normal cometer errores), los manuales Español Moderno y similares poseen unos contenidos léxicos y gramaticales que han quedado obsoletos. La nueva gramática española difiere mucho de la gramática tratada en estos manuales, y las dificultades empiezan cuando los alumnos comienzan a cuestionarse la veracidad de los contenidos que les enseñan los profesores extranjeros. Cuando un profesor extranjero explica y algunos de estos contenidos son diferentes de los del manual chino, los alumnos empezarán a cuestionar la información del profesor, y tendrán la convicción de que toda la información plasmada en los manuales chinos carece de inadecuación o error alguno. También es verdad que, poco a poco, el alumnado toma conciencia y está desarrollando una visión más crítica al respecto; sin embargo, aún no se aprecian cambios relevantes en esta conducta.

Con respecto a la metodología, la empleada en estos manuales es la estructuralista (aunque no podemos considerar que esta metodología se aplique en estado puro o en el cien por cien de los casos). Este método de enseñanza influye negativamente en las capacidades del alumno para razonar cualquier información. Otro problema de los manuales chinos está en el nivel de los textos. Los contenidos en este estadio no solamente no se adecuan a los estándares de enseñanza internacionales. Ni siquiera se aprecia un acercamiento. El nivel de los textos es, desde el principio, estratosférico. Por este motivo, los alumnos recitan los textos una y otra vez para memorizarlos, porque, debido a la falta de comprensión de los contenidos, no logran entender el significado de las muestras. Desde el comienzo de su estudio del español, el alumno se enfrenta a estos problemas y, en muchas ocasiones, se aprecian aprendientes frustrados, "quemados" intelectualmente a causa de la incomprensión. Es aquí cuando el sujeto alcanza un estado de pasividad $y$, si sumamos los contenidos, la incomprensión y la falta de metodología comunicativa, además de la carencia de una buena metodología de estudio, poco a poco el alumno "se aleja y se pierde" en el aprendizaje de la lengua.

Finalmente, es necesario decir que no todo son amarguras, pues también debemos destacar que, entre los aspectos positivos que tienen estos manuales, se encuentra la realidad hispanoamericana. Podemos ver contenidos relacionados con muchos otros países de habla hispana, que abarcan terrenos culturales, geográficos, literarios e históricos.

Los manuales extranjeros comprenden un enfoque ecléctico e incluso adaptado, en ocasiones, a las necesidades de los alumnos chinos (aunque algunas veces los contenidos no consiguen motivar al alumnado); no obstante, pese a las ayudas que ofrecen, no logran funcionar del todo, y esto se debe a que estos aprendientes no han desarrollado las competencias adecuadas para aceptar un enfoque comunicativo.

\section{Conclusiones}

Tras las muestras que hemos obtenido a la largo de nuestra investigación, podemos afirmar que, con respecto a la metodología, la empleada en los manuales chinos no es otra que la estructuralista; sin embargo, este tipo de estructuralismo es más notable que el existente en otros países asiáticos, pues, entre las consecuencias y los efectos derivados de este enfoque pedagógico, se encuentra la merma total en el uso del razonamiento por parte del aprendiente. Solamente se desarrolla un aprendizaje memorístico, sin aplicaciones contextuales ni posibilidad de ra- 
zonamiento. Todo está concebido para indicar al alumno qué debe estudiar y, por consiguiente, memorizar sistemáticamente.

El aprendiente, por tanto, sistematiza todo, intenta buscar equivalencias exactas con su propio idioma, y, por tanto, traduce o interpreta automáticamente (palabra por palabra) sus pensamientos en chino. El alumno no puede, entonces, comprender o conocer el trasfondo o los principios del razonamiento español. Podemos decir que el aprendiente no logra entrar en la "cosmovisión lingüística hispana”. Con esto, queremos decir que el sujeto no logra "ver" la lengua española como nosotros la vemos, sino desde la realidad china.

\section{Referencias bibliográficas}

Aguirre Beltrán, Blanca, 2004: Análisis de necesidades y diseño curricular, Universidad Complutense de Madrid.

Alcoholado, Antonio y Mariona Anglada, 2011: "Usar las manos: elaboración de material didáctico para asignaturas extralingüísticas en China”, SinOELE 5, 31-59.

Alcoholado, Antonio, 2012: "La sílaba como unidad estructural: propuesta de actividades para principiantes sinófonos de ELE", SinoELE 6, 71-83.

Anglada Escudé, Mariona y Xiao Zhang, 2012: "El método Español Moderno (vol. I): revisión normativa y de su enfoque ortográfico y gramatical", sinOELE 6 [disponible en http://www.sinoele.org/ index.php/49-revista-1/sinoele-6].

Bega González, Michel René, 2014: Enseñar lengua española en China: análisis de los problemas lingüísticos, comunicativos y metodológicos. Tesis doctoral, Universidad de Las Palmas de Gran Canaria, España.

Breen, M. y C. N. Candling, 1980: "The essentials of a communicative curriculum in language teaching", Applied Linguistics 1/2, 89-112.
Caceres Lorenzo, María Teresa y Yaiza Santana AlvaraDo, 2014: "La rúbrica de un taller de escritura para enseñar a sinohablantes en el contexto universitario europeo: estudio de caso", Porta Linguarum 21, 227-244.

Canale, Michael: "De la competencia comunicativa a la pedagogía comunicativa del lenguaje" [disponible en http://cvc.cervantes.es/ensenanza/biblioteca_ele/antologia_didactica/enfoque_comunicativo/canaleo1.htm. Consultada el 16/04/2014].

Celce-Murcia, Marianne y Elite Olshtain, 2000: Discourse and context in language teaching. A Guide for Language Teachers, Cambridge: University Press.

Cenoz Iragul, Jasone, 2004: El concepto de competencia comunicativa en Vademécum para la formación de profesores: Enseñar español como segunda lengua (L2) / lengua extranjera (LE), Madrid: SGEL, 2004.

Cenoz Iragul, Jasone. "El concepto de competencia comunicativa" [disponible en http://cvc.cervantes.es/Ensenanza/biblioteca_ele/antologia_didactica/enfoque_comunicativo/cenozor.htm. Consultada el 16/04/2014].

Centro Virtual Cervantes (CVC): "Diccionario de términos clave de ELE" [disponible en http://cvc. cervantes.es/ensenanza/biblioteca_ele/diccio_ ele/indice.htm. Consultada el 30/03/2015].

De Miguel Díaz, Mario, 2004: Modalidades de enseñanza centradas en el desarrollo de competencias. Orientaciones para promover el cambio metodológico en el Espacio Europeo de Educación Superior, Oviedo: Servicio de Publicaciones de la Universidad de Oviedo.

Eluss, Rod, 1994: The Study of Second Language Acquisition, Oxford: Oxford University Press.

García Santana-Cecilia, Álvaro: "Lengua y comunicación: tres décadas de cambio" [disponible en http://cvc.cervantes.es/Ensenanza/biblioteca_ 
ele/antologia_didactica/enfoque_comunicativo/garcia01.htm. Consultada el 16/04/2014].

Hawkins, Eric, 1984: Awareness of Language: An Introduction, Cambridge: Cambridge University Press.

Hu, Guangwei, 2002: "Potential Cultural Resistance to Pedagogical Imports: The Case of Communicative Language Teaching in China, en Language", Culture and Curriculum 15, 93-105.

LitTlewood, W., 1981: Communicative Language Teaching, Cambridge: C. U. P.

Martín Martín, José Miguel, 2003: "Sobre lo contrastivo y el conocimiento lingüístico previo en la enseñanza del español" en La competencia lingüística en el aprendizaje del español como lengua extranjera, Madrid: Edinumen.

Penner, Janice, 1995: "Change and Conflict: Introduction of the Communicative Approach in China", TESL Canada Journal 12 [disponible en http:// www.teslcanadajournal.ca/index.php/tesl/article/view/649/480. Consultada el 12/06/13]

Ruiz Campillo, José P. y Gracia Lozano López: "Hacia una evaluación interna de la tarea comunicativa" [disponible en http://cvc.cervantes.es/Ensenanza/biblioteca_ele/antologia_didactica/enfoque_comunicativo/campilloo1.htm. Consultada el 16/04/2014].

Sanchez Griñan, Alberto, 2009: "Estrategias de aprendizaje de alumnos chinos de español", MarCOELE 8 .

Sánchez Griñán, Alberto José: "Dificultades del enfoque comunicativo en China" [disponible en http://congresoele.net/biblioteca/index. php?option=com_content\&task=view\&id $=115 \&$ l temid=48. Consultada el 29/01/2014].

Sánchez Griñan, Alberto José, 2008: Enseñanza y aprendizaje de español como lengua extranjera en China. Retos y posibilidades del enfoque comunicativo, Universidad de Murcia.
Sanchez Griñán, Alberto José y otros: "Contextos de enseñanza y aprendizaje de ELE en el ámbito sinohablante" [disponible en http://www.sinoele. org/index.php?option=com_content\&view=artic le\&id=123\&ltemid=103\&lang=zh-CN. Consultada el 21/06/2013].

Sanchez, A. 1997: Los métodos en la enseñanza de idiomas. Evolución histórica y análisis didáctico, Madrid: SGEL.

Swan, Michael. "Una Mirada crítica al enfoque comunicativo (1)" [disponible en http://cvc.cervantes.es/Ensenanza/biblioteca_ele/antologia_didactica/enfoque_comunicativo/swano1_l.htm. Consultada el 16/04/2014.]

Swan, Michael: "Una Mirada crítica al enfoque comunicativo (2)" [disponible en http://cvc.cervantes.es/Ensenanza/biblioteca_ele/antologia_didactica/enfoque_comunicativo/swanos_II.htm. Consultada el 16/04/2014].

Vacas Hermida, Asunción, 2004: "El enfoque por tareas", Tierra de Nadie 9 [disponible en http:// www.tierradenadie.de/articulos/enfoqueportareas.htm. Consultada el 10/04/2013].

WANG, Ting y Leah Moore, 2007: "Exploring Learning Style Preferences of Chinese Postgraduate Students in Australian Transnational Programs", International Journal of Pedagogies and Learning 3(2), 31-41.

Xu, Yang, 2010: English Language Teaching, CCSE.

Zabalza Beraza, Miguel Ángel, 2007: Competencias docentes del profesorado universitario: Calidad y desarrollo profesional, Madrid: Narcea. 